\title{
Electrical detection of magnetic skyrmions by tunnelling non-collinear magnetoresistance
}

\author{
Christian Hanneken 1 , Fabian Otte 2 , André Kubetzka1*, Bertrand Dupé2, Niklas Romming1, \\ Kirsten von Bergmann ${ }^{1 \star}$, Roland Wiesendanger' and Stefan Heinze ${ }^{2}$
}

\begin{abstract}
Magnetic skyrmions are localized non-collinear spin textures with a high potential for future spintronic applications ${ }^{1-12}$. Skyrmion phases have been discovered in a number of materials $s^{9,11}$ and a focus of current research is to prepare, detect and manipulate individual skyrmions for implementation in devices ${ }^{6-8}$. The local experimental characterization of skyrmions has been performed by, for example, Lorentz microscopy ${ }^{3}$ or atomic-scale tunnel magnetoresistance measurements using spin-polarized scanning tunnelling microscopy $4,7,12$. Here we report a drastic change of the differential tunnel conductance for magnetic skyrmions that arises from their non-collinearity: mixing between the spin channels locally alters the electronic structure, which makes a skyrmion electronically distinct from its ferromagnetic environment. We propose this tunnelling non-collinear magnetoresistance as a reliable all-electrical detection scheme for skyrmions with an easy implementation into device architectures.
\end{abstract}

In magnetic skyrmions, the magnetization in the centre is opposite to that of the surrounding ferromagnetic (FM) background. Skyrmions are stabilized against collapse by the DzyaloshinskiiMoriya interaction ${ }^{13-15}$, which arises because of the spin-orbit interaction in systems with broken inversion symmetry and imposes a unique rotational sense on the spin structure. Such a particle-like magnetic skyrmion is distinct from the FM state because of its non-trivial topology, and its topological charge can be used as bit of information 5,6 . For the readout of information encoded in magnetic states, various magnetoresistive effects are exploited in current technical devices and sensors. Whereas the giant magnetoresistance $(\mathrm{GMR})^{16,17}$ and tunnel magnetoresistance (TMR) $)^{18,19}$ occur when two magnetic layers are involved (Fig. 1a), the (tunnelling) anisotropic magnetoresistance (T)AMR ${ }^{20-22}$ (Fig. 1b) originates from the intrinsic material properties caused by spin-orbit coupling and typically amounts to only a few percent. In transport measurements through non-collinear structures (for example, domain walls), the total resistance deviates from that of the FM case $\mathrm{c}^{23-26}$. However, as a result of the averaging nature of these measurements, discrimination between different contributing effects is difficult ${ }^{25,26}$. In particular, the extent to which the non-collinearity itself is a source of magnetoresistance is unclear (Fig. 1c).

For a spatially resolved disentanglement of different contributions to magnetoresistance we chose, as a model system, magnetic skyrmions (Fig. 2a) in the PdFe atomic bilayer on $\operatorname{Ir}(111)^{7,10,12}$, which have been studied previously using spin-polarized scanning tunnelling microscopy (STM). This method ${ }^{27}$ resembles the TMR in STM geometry, in which a magnetic tip is separated from the magnetic sample by an insulating vacuum barrier. Figure $2 \mathrm{~b}$ shows an overview of a PdFe/Ir(111) sample in the skyrmion phase $(B=+1.8 \mathrm{~T})$ measured with an STM tip that is not spin polarized (that is, the TMR contribution vanishes). Nevertheless, the skyrmions in the PdFe islands are imaged as dark circular entities in the map of differential tunnel conductance $\mathrm{d} I / \mathrm{d} U$, where $I$ is the tunnel current and $U$ is the sample bias voltage. The enlarged $\mathrm{d} I / \mathrm{d} U$ image (Fig. 2c) shows two skyrmions at $B=-2.5 \mathrm{~T}$ and the line profile across one of them (Fig. 2c, inset) shows that the $\mathrm{d} I / \mathrm{d} U$ signal changes gradually from the FM region to about half of its value at the skyrmion centre. The question of the physical origin of the signal change arises because a TMR is not present, as an unpolarized tip is used, and neither is a TAMR contribution expected, because the centre of the skyrmion is antiparallel to the spins in the FM background (see also Supplementary Section 1).

The energy-resolved $\mathrm{d} I / \mathrm{d} U$ signal measured by scanning tunnelling spectroscopy can be interpreted as the sample's local density of states (LDOS) in the vacuum ${ }^{28}$, which correlates with the electronic band structure. Figure $2 \mathrm{~d}$ demonstrates that the local electronic properties of the skyrmion centre deviate significantly from those of the FM background: the spectrum of the FM state has a peak at about $+0.7 \mathrm{~V}$, whereas the centre of the skyrmion exhibits two peaks at about +0.5 and $+0.9 \mathrm{~V}$. We propose that this difference originates from the non-collinearity of the spin structure in the skyrmion.

To validate this hypothesis we performed an experiment in which we changed the degree of non-collinearity in a controlled fashion by varying the external magnetic field. The size and shape of a skyrmion in PdFe has been studied as a function of the applied field ${ }^{12}$, and the polar angle $\theta(d)$ of the magnetization within a skyrmion is plotted in Fig. $3 \mathrm{a}$ as a function of the lateral
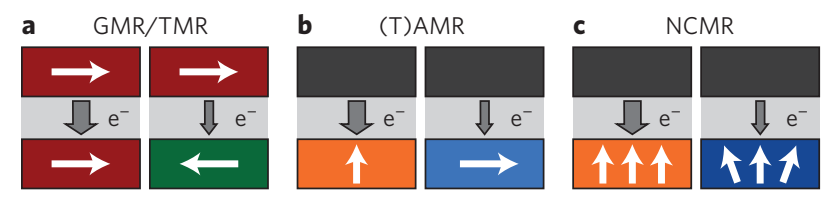

Figure 1 | Magnetoresistive effects in planar junctions. a, Sketch of the GMR and TMR effect, in which two magnetic electrodes are separated by a non-magnetic metal or an insulator, respectively; in the sketch the conductance is larger for the parallel than for the antiparallel magnetization alignment. b. The (T)AMR effect does not require a magnetic sensor electrode and arises from intrinsic spin-orbit coupling within the magnetic layer; the conductance for the out-of-plane (up or down) magnetization is different to that of the in-plane magnetization. c, The NCMR proposed in this work leads to different conductance signals for collinear compared with non-collinear magnetic states.

'Department of Physics, University of Hamburg, Jungiusstrasse 11, Hamburg 20355, Germany. ${ }^{2}$ Institute of Theoretical Physics and Astrophysics, ChristianAlbrechts-Universität zu Kiel, Leibnizstrasse 15, Kiel 24098, Germany. ${ }^{\star}$ e-mail: kubetzka@physnet.uni-hamburg.de; kbergman@physnet.uni-hamburg.de 

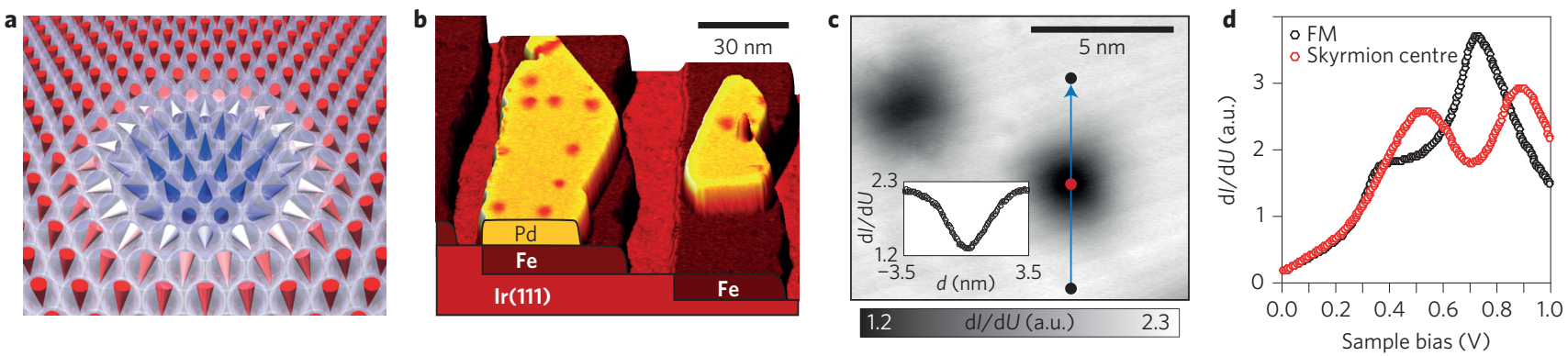

Figure 2 | Individual skyrmions in PdFe/Ir(111). a, Sketch of a magnetic skyrmion; cones represent the magnetization direction. $\mathbf{b}$, Perspective view of an STM constant-current image, colour-coded with the $\mathrm{d} / / \mathrm{d} U$ signal; yellow areas indicate PdFe and red circular entities are magnetic skyrmions; $\mathrm{Pd}$ is in a hexagonal close-packed stacking arrangement on the face-centred cubic stacked Fe on $\operatorname{Ir}(B=+1.8 \mathrm{~T}, U=+0.7 \mathrm{~V}, I=1 \mathrm{nA}, T=8 \mathrm{~K})$. c, $\mathrm{Closer}$ view $\mathrm{d} l / \mathrm{d} U$ map of two skyrmions; the inset presents a profile along the arrow $(B=-2.5 \mathrm{~T}, U=+0.7 \mathrm{~V}, I=1 \mathrm{nA}, T=4 \mathrm{~K})$. $\mathrm{d}, \mathrm{d} l / \mathrm{d} U$ tunnel spectra in the centre of a skyrmion (red) and outside the skyrmion in the FM background (black) $(B=-2.5 \mathrm{~T}, T=4 \mathrm{~K}$; stabilization parameters, $U=-1 \mathrm{~V}, \mathrm{I}=1 \mathrm{nA})$. a.u., arbitrary units.
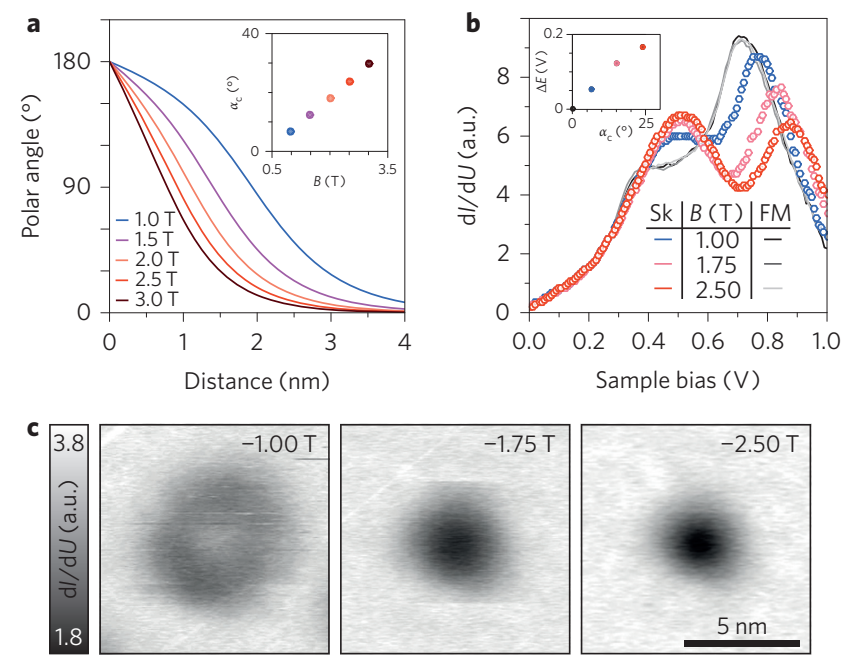

Figure 3 | Magnetic field-dependent properties of an individual skyrmion. a, Skyrmion profiles for different magnetic field values, plotted as polar angle $\theta$ of the magnetization versus distance from the skyrmion centre (obtained from fits to spin-polarized STM measurements ${ }^{12}$ ). Inset: evolution of the angle between a central spin of a skyrmion and its neighbours, $\alpha_{\mathrm{C}^{\prime}}$ with the external magnetic field $B . \mathbf{b}, \mathrm{d} l / \mathrm{d} U$ tunnel spectra measured with a $W$ tip in the centre (Sk) and outside (FM) of an individual skyrmion at different magnetic field values ( $T=8 \mathrm{~K}$; stabilization parameters $U=-0.3 \mathrm{~V}, I=0.2 \mathrm{nA}$ ). Inset: evolution of the energy shift of the high-energy peak with respect to the FM state, $\Delta E$, as a function of the angle between spins in the centre of the skyrmion, $\alpha_{\mathrm{c}}$ (see the inset in $\mathbf{a}$ for the relation between $\alpha_{\mathrm{c}}$ and $B$ ). c, Corresponding laterally resolved $\mathrm{d} / / \mathrm{d} U$ maps $(U=+0.7 \mathrm{~V}, I=1 \mathrm{nA}, T=8 \mathrm{~K})$.

distance $d$ from its centre for several magnetic field values. We relate the degree of non-collinearity in the centre of a skyrmion with the angle $\alpha_{c}$ between a central atom and its neighbouring spins, and find that $\alpha_{c}$ scales linearly with $B$ (inset of Fig. 3a). Figure $3 \mathrm{~b}$ displays spectra taken at the centre of one skyrmion at different applied fields, as indicated, together with reference spectra of the FM background. One can clearly see a systematic shift of the higher-energy peak with the applied field. The peak shift $\Delta E$ with respect to the peak of the FM state is roughly linear with $\alpha_{c}$ (inset of Fig. 3b), which corroborates our proposal of an effect of the local magnetic non-collinearity on the electronic properties. The laterally resolved $\mathrm{d} I / \mathrm{d} U$ maps at the FM peak energy in Fig. 3c show how the maximum of non-collinearity moves from the rim of the skyrmion to its centre with increasing magnetic field, in agreement with the skyrmion profiles in Fig. 3a.
For the FM state, the experimental $\mathrm{d} I / \mathrm{d} U$ spectra (Figs $2 \mathrm{~d}$ and $3 \mathrm{~b}$ ) are in good agreement with the vacuum LDOS calculated by density functional theory $(\mathrm{DFT})^{10}$ (Fig. 4a). The vacuum LDOS is typically dominated by states close to the $\bar{\Gamma}$ point. A detailed analysis of the spin-resolved band structure and LDOS (Supplementary Sections 2 and 3) reveals that the sharp peak at about $+0.9 \mathrm{eV}$ stems from the minority $d$ states, whereas the step-like LDOS of the majority spin channel is caused by bands of $s$ and $p$ character.

In a non-collinear spin structure, there is a mixing between the two spin channels that results in a change of the band structure and the $\operatorname{LDOS}^{29}$. This is seen in DFT calculations for the spin spiral phase (Supplementary Sections 2 and 3), which are in agreement with the corresponding experimental data (Supplementary Section 4). To capture the key physics of this band mixing for two-dimensional (2D) localized skyrmions and to include the skyrmion profiles ${ }^{12}$ (Fig. 3a) we use a tight-binding (TB) model. The corresponding Hamiltonian at every atom site is given by

$$
H_{0}=\left(\begin{array}{cc}
\epsilon_{\uparrow} & 0 \\
0 & \epsilon_{\downarrow}
\end{array}\right)
$$

where $\epsilon_{\uparrow}, \epsilon_{\downarrow}$ are the on-site energies of the two states. Based on DFT for the FM state, we describe the electronic states of PdFe/ $\operatorname{Ir}(111)$, which dominate the vacuum LDOS, by using a majority band with a hopping parameter $t_{\uparrow}=-0.5 \mathrm{eV}$, and a minority band with $t_{\downarrow}=+0.09$ and $\epsilon_{\uparrow}-\epsilon_{\downarrow}=3.1 \mathrm{eV}$, as depicted in green and red in Fig. 4b. The corresponding spin-resolved LDOS in the vacuum for the FM state is qualitatively very similar to that obtained by DFT calculations ${ }^{10}$ (compare Fig. 4a,c) and a similar agreement is obtained for the spin spiral states (Supplementary Section 3). The non-collinearity within the skyrmion leads to a mixing between the majority and the minority spin channels and the hopping between adjacent atomic sites can be described by the matrix

$$
V\left(\alpha_{i j}\right)=\left(\begin{array}{cc}
t_{\uparrow} \cos \left(\alpha_{i j} / 2\right) & -t_{\uparrow \downarrow} \sin \left(\alpha_{i j} / 2\right) \\
t_{\downarrow \uparrow} \sin \left(\alpha_{i j} / 2\right) & t_{\downarrow} \cos \left(\alpha_{i j} / 2\right)
\end{array}\right)
$$

where $\alpha_{i j}$ is the angle between the spins on neighbouring sites $i$ and $j$ and $t_{\uparrow \downarrow}=-t_{\downarrow \uparrow}$ describes the nearest-neighbour hopping matrix element between the two states.

Before solving this TB model for a realistic skyrmion profile, it is instructive to study the effect of the spin mixing in a simplified way. We assume that the matrix $V\left(\alpha_{i j}\right)$ is the same for all atom sites by fixing all $\alpha_{i j}$ to the same angle $\alpha$ and thus obtain a 

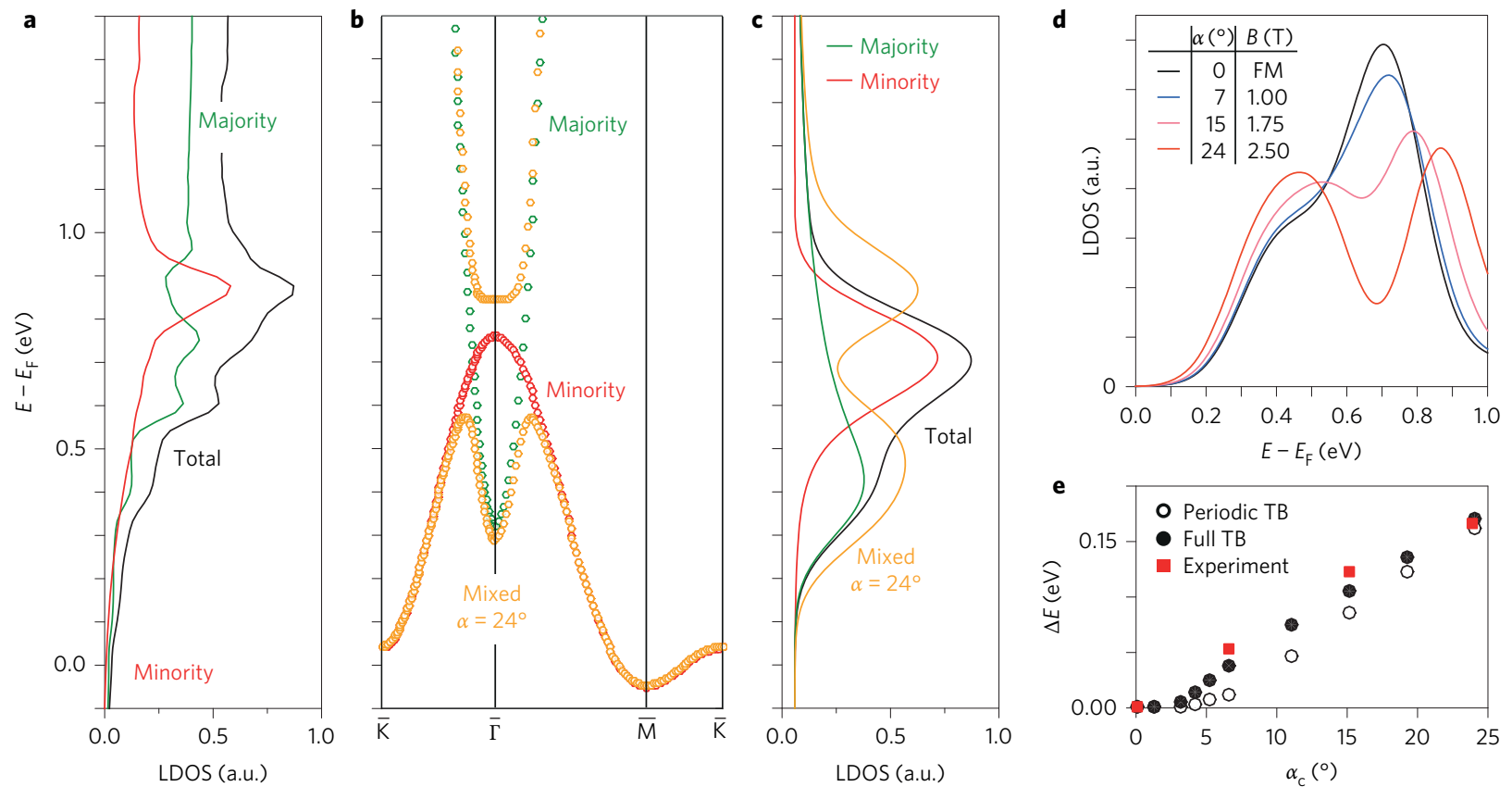

Figure 4 | DFT and TB calculations. a, Vacuum LDOS calculated from DFT for the FM state at a distance of about $12 \AA$ from the surface. The black line is the total vacuum LDOS and the green and red lines represent the majority and minority spin channels, respectively. $\mathbf{b}, \mathbf{c}$, Periodic TB model band structure (b) and the corresponding vacuum LDOS for the FM state (c) with majority (green) and minority (red) spins and for $\alpha=24^{\circ}$ between nearest-neighbour spins (orange, see text for the details). d, Vacuum LDOS from the periodic TB model at different angles $\alpha$; the data can be correlated to a magnetic field in which the angle between neighbouring magnetic moments is realized in the centre of a skyrmion (see the inset of Fig. 3a). e, Peak shift of the higher-energy peak with respect to the FM state for the periodic and the full TB calculations in the centre of the skyrmion, and the experimental data from the inset of Fig. 3b for comparison.

periodic system with a well-defined band structure. Figure $4 \mathrm{~b}$ shows that a non-zero value of $\alpha$ (orange) leads to the formation of a gap in the band structure near the $\bar{\Gamma}$ point at which the bands were crossing formerly. This leads to the emerging two-peak structure in the vacuum LDOS (see Fig. 4c), in good agreement with the experimental $\mathrm{d} I / \mathrm{d} U$ spectra taken at the skyrmion centre (Fig. 2d).

The energy splitting between the peaks in the vacuum LDOS increases with the angle $\alpha$ between adjacent spins, as demonstrated in Fig. $4 \mathrm{~d}$, and $\alpha$ can be correlated to the magnetic-field dependent nearest-neighbour angle in the centre of a skyrmion $\alpha_{c}$ (see the Fig. 3a inset). We include the experimentally determined magnetization profile of a skyrmion ${ }^{12}$ (Fig. 3a) by now choosing $\alpha_{i j}$ in the matrix $V\left(\alpha_{i j}\right)$ differently for all nearest-neighbour sites of the hexagonal lattice according to the local spin orientation and solve this full nearest-neighbour TB model numerically (Methods). Qualitatively, the LDOS at the centre of the skyrmion behaves as in the periodic TB model. However, taking into account the whole spin structure of the skyrmion leads to a larger shift of the highenergy peak with $\alpha_{c}$, as shown in Fig. 4e. We attribute the better quantitative agreement with the experimental data to the fact that the local variation of the electronic structure within the skyrmion is captured within the full TB model.

Another means to study the effect of non-collinearity is to use the spatial-resolution capabilities of STM to investigate the electronic properties within one skyrmion as a function of distance to the centre. As anticipated from the plot in Fig. 3a, the spectra of a skyrmion at $B=-2.5 \mathrm{~T}$ show a continuous variation of the peak position from the FM spectrum to the spectrum in the centre of the skyrmion (bottom and top spectra in Fig. 5a, respectively). The laterally resolved vacuum LDOS from the full TB model (Fig. 5b) also shows a peak shift, in very good agreement with the experiment. To further analyse the effect of non-collinearity on the electronic properties for $\mathrm{PdFe} / \mathrm{Ir}(111)$, we extract the energy shift $\Delta E$ of the high-energy peak with respect to the FM spectrum for both the experimental data and the calculations and plot it against the lateral distance to the skyrmion centre (Fig. 5c). Whereas the evolution of $\Delta E$ across a skyrmion at $B=-2.5 \mathrm{~T}$ has a maximum at the centre, the maximum $\Delta E$ for a skyrmion at $B=-1 \mathrm{~T}$ (Fig. $5 \mathrm{~d}$ ) is much smaller and lies on a circle around the skyrmion centre with a radius of about $1.5-2 \mathrm{~nm}$, in agreement with the results shown in Fig. 3a,c.

Although in a local STM measurement we obtain a signal change caused by the non-collinear magnetoresistance (NCMR) of up to $100 \%$ for the small skyrmions at $-2.5 \mathrm{~T}$ (inset of Fig. $2 \mathrm{c}$ ), the net NCMR will be reduced in spatially averaging planar tunnel junctions. In such a geometry, which is more relevant for applications, large angles in the centre of a skyrmion are not required or even advantageous, but instead one can profit from an increased area of non-collinear spin arrangements. In our system, for instance, the NCMR of a skyrmion in a $100 \mathrm{~nm}^{2}$ junction would be twice as large at $-1 \mathrm{~T}$ compared with that at $-2.5 \mathrm{~T}$, because the smaller local signal is overcompensated by the larger skyrmion size (compare Fig. 5c,d).

Our work demonstrates the impact of the degree of non-collinearity of a spin structure on the corresponding differential tunnel conductance. In contrast to the GMR, TMR and (T)AMR, in which the signal depends on the local magnetization direction (Fig. 1), the NCMR is sensitive to the local magnetic environment and does not require a magnetic electrode for detection. As it originates from the spin mixing of bands of opposite spin channels, which is a general effect in non-collinear spin structures that results in a change of the local electronic structure, we anticipate that this NCMR effect occurs in a wide range of magnetic materials and is not limited to the tunnel regime. In particular, we propose to use NCMR for the detection of nanoscale skyrmions with a nonmagnetic electrode in racetrack-type spintronic devices ${ }^{6,30}$ with planar tunnel junctions as stationary read heads. 

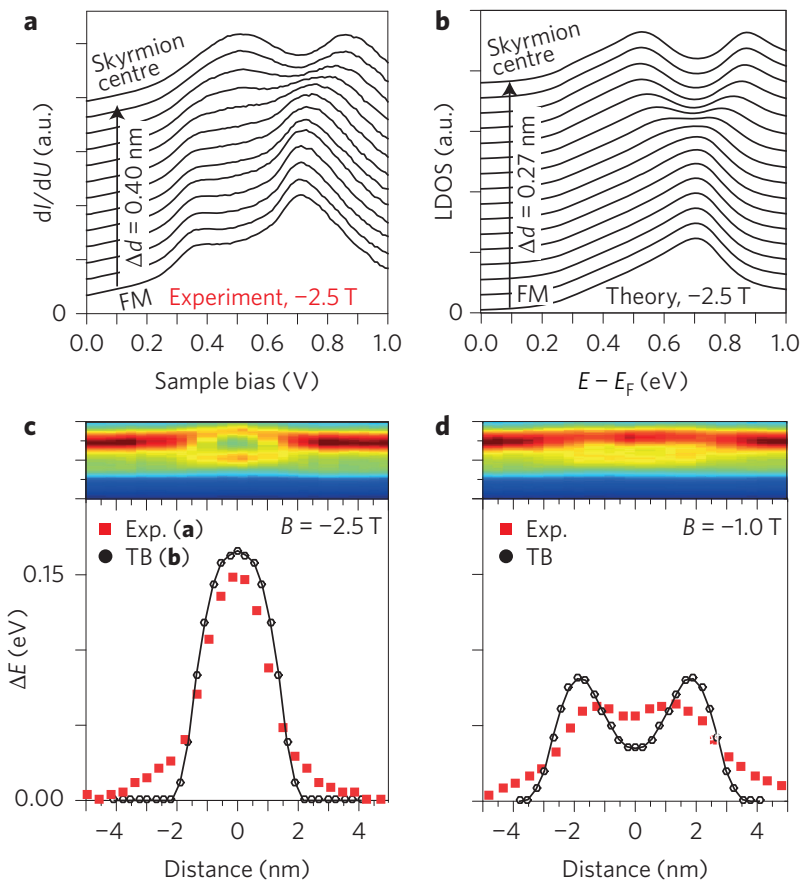

Figure $\mathbf{5}$ | Spatial variation of the differential tunnel conductance and the calculated vacuum LDOS within a skyrmion. a, Experimental $\mathrm{d} / \mathrm{d} U$ tunnel spectra measured with a $W$ tip at different lateral positions $d$ of the skyrmion at a field of $B=-2.5 \mathrm{~T}$; the FM spectrum is presented at the bottom and the spectra towards the skyrmion centre (top) are vertically offset for clarity. $\mathbf{b}$, Corresponding plot of the vacuum LDOS for a magnetic skyrmion calculated from the full TB model. c, Shift of the high-energy peak as a function of distance to the skyrmion centre extracted from the experimental data (red rectangles) and the full TB model (black circles); the inset at the top shows a colour-coded plot of the data of $\mathbf{a}$ with energy (vertical) and lateral (horizontal) resolution; red and yellow colours indicate the peak positions. $\mathbf{d}$, Same as $\mathbf{c}$ but for $B=-1 \mathrm{~T}$. The maximum of $\Delta E$ is now located off-centre at $|d|=1.5-2.0 \mathrm{~nm}$

\section{Methods}

Methods and any associated references are available in the online version of the paper.

\section{Received 12 May 2015; accepted 20 August 2015;} published online 5 October 2015

\section{References}

1. Bogdanov, A. N. \& Yablonskii, D. A. Thermodynamically stable 'vortices' in magnetically ordered crystals. The mixed state of magnets. Sov. Phys. JETP 68, 101-103 (1989)

2. Mühlbauer, S. et al. Skyrmion lattice in a chiral magnet. Science 323, 915-919 (2009).

3. Yu, X. Z. et al. Real space observation of a two-dimensional skyrmion crystal. Nature 465, 901-904 (2010).

4. Heinze, S. et al. Spontaneous atomic-scale magnetic skyrmion lattice in two dimensions. Nature Phys. 7, 713-718 (2011)

5. Kiselev, N. S., Bogdanov, A. N., Schäfer, R. \& Rößler, U. K. Chiral skyrmions in thin magnetic films: new objects for magnetic storage technology? J. Phys. D 44, 392001 (2011).

6. Fert, A., Cros, V., \& Sampaio, J. Skyrmions on the track. Nature Nanotech. 8, 152-156 (2013).

7. Romming, N. et al. Writing and deleting single skyrmions. Science 341, 636-639 (2013).

8. Sampaio, J., Cros, V., Rohart, S., Thiaville, A. \& Fert, A. Nucleation, stability and current-induced motion of isolated magnetic skyrmions in nanostructures. Nature Nanotech. 8, 839-844 (2013).
9. Nagaosa, N. \& Tokura, Y. Topological properties and dynamics of magnetic skyrmions. Nature Nanotech. 8, 899-911 (2013).

10. Dupé, B., Hoffmann, M., Paillard, C. \& Heinze, S. Tailoring magnetic skyrmions in ultra-thin transition metal films. Nature Commun. 5, 4030 (2014).

11. von Bergmann, K., Kubetzka, A., Pietzsch, O. \& Wiesendanger, R. Interfaceinduced chiral domain walls, spin spirals and skyrmions revealed by spinpolarized scanning tunneling microscopy. J. Phys. Condens. Matter 26, 394002 (2014)

12. Romming, N., Kubetzka, A., Hanneken, C., von Bergmann, K. \& Wiesendanger, R. Field-dependent size and shape of single magnetic skyrmions. Phys. Rev. Lett. 114, 177203 (2015).

13. Dzyaloshinskii, I. E. Thermodynamic theory of 'weak' ferromagnetism in antiferromagnetic substances. Sov. Phys. JETP 5, 1259-1262 (1957).

14. Moriya, T. Anisotropic superexchange interaction and weak ferromagnetism. Phys. Rev. 120, 91-98 (1960).

15. Fert, A. \& Levy, P. A. Role of anisotropic exchange interactions in determining the properties of spin glasses. Phys. Rev. Lett. 44, 1538-1541 (1980).

16. Baibich, M. N. et al. Giant magnetoresistance of $(001) \mathrm{Fe} /(001) \mathrm{Cr}$ magnetic superlattices. Phys. Rev. Lett. 61, 2472-2475 (1988).

17. Binasch, G., Grünberg, P., Saurenbach, F. \& Zinn, W. Enhanced magnetoresistance in layered magnetic structures with antiferromagnetic interlayer exchange. Phys. Rev. B 39, 4828 (1989).

18. Jullière, M. Tunneling between ferromagnetic films. Phys. Lett. A 54, 225-226 (1975).

19. Wiesendanger, R., Güntherodt, H.-J., Güntherodt, G., Gambino, R. J. \& Ruf, R Observation of vacuum tunneling of spin-polarized electrons with the scanning tunneling microscope. Phys. Rev. Lett. 65, 247 (1990).

20. McGuire, T. R. \& Potter, R. I. Anisotropic magnetoresistance in ferromagnetic 3D alloys. IEEE Trans. Magn. 11, 1018-1038 (1975).

21. Bode, M. et al. Magnetization-direction dependent local electronic structure probed by scanning tunneling spectroscopy. Phys. Rev. Lett. 89, 237205 (2002).

22. Gould, C. et al. Tunneling anisotropic magnetoresistance: a spin-valve-like tunnel magnetoresistance using a single magnetic layer. Phys. Rev. Lett. 93, 117203 (2004)

23. Levy, P. M. \& Zhang, S. Resistivity due to domain wall scattering. Phys. Rev. Lett. 79, 5110-5113 (1997)

24. Kent, A. D., Yu, J., Rüdiger, U. \& Parkin, S. S. P. Domain wall resistivity in epitaxial thin film microstructures. J. Phys. Condens. Matter 13, 461-468 (2001)

25. Marrows, C. H. \& Dalton, B. C. Spin mixing and spin-current asymmetry measured by domain wall magnetoresistance. Phys. Rev. Lett. 92, 97206 (2004).

26. Seemann, K. M. et al. Disentangling the physical contributions to electrical resistance in magnetic domain walls: a multiscale study. Phys. Rev. Lett. 108, 77201 (2012).

27. Wiesendanger, R. Spin mapping at the nanoscale and atomic scale. Rev. Mod. Phys. 81, 1495-1550 (2009).

28. Tersoff, J. \& Hamann, D. Theory of the scanning tunneling microscope. Phys. Rev. B 31, 805-813 (1985).

29. Sandratskii, L. M. Noncollinear magnetism in itinerant-electron systems: theory and applications. Adv. Phys. 47, 91-160 (1998).

30. Parkin, S. S. P., Hayashi, M. \& Thomas, L. Magnetic domain-wall racetrack memory. Science 320, 190-194 (2008).

\section{Acknowledgements}

C.H, A.K, N.R., K.v.B., R.W., S.H. and B.D. acknowledge financial support from the Deutsche Forschungsgemeinschaft via GrK 1286, SFB 668 and project DU1489/2-1. S.H. and B.D. thank the North-German Supercomputing Alliance (HLRN) for providing computational resources. We thank P. Mavropoulos, Y. Mokrousov, G. Bihlmayer and A. Kobs for discussions.

\section{Author contributions}

C.H. performed the measurements, C.H., K.v.B. and A.K. analysed the experimental data C.H., F.O. and A.K. prepared the figures, and K.v.B., A.K. and S.H. wrote the manuscript. B.D. performed the DFT calculations, F.O. and S.H. devised the TB model, F.O. performed the TB calculations and F.O., B.D. and S.H. analysed the calculations. All the authors discussed the results and contributed to the manuscript.

\section{Additional information}

Supplementary information is available in the online version of the paper. Reprints and permissions information is available online at www.nature.com/reprints. Correspondence and requests for materials should be addressed to K.v.B. and A.K.

\section{Competing financial interests}

The authors declare no competing financial interests. 


\section{Methods}

We apply the Greens-function technique $e^{31}$ to solve numerically the two-band nearest-neighbour TB model given by equations (1) and (2). We use the hopping parameters given in the text and solve the TB model for a $2 \mathrm{D}$ hexagonal lattice of $(30 \times 30)$ atom sites with periodic boundary conditions in the $y$ direction and semiinfinite FM lattices in the $x$ direction, which is the close-packed direction. The local spin-quantization axis on the atomic lattice, which determines the angle $\alpha_{i j}$ for nearest-neighbour sites $i$ and $j$ in equation (2), is chosen according to the skyrmion profile $^{12} \theta(d)$ (see Fig. 3a). We account for the broadening of bands that results from hybridization with the metal substrate by adding an imaginary term -i - to the diagonal elements of our Hamiltonian, with $\gamma=0.1 \mathrm{eV}$. The value of $t_{\downarrow \uparrow}=0.17 \mathrm{eV}$ was adapted to the experimentally observed peak shift. We obtained the vacuum LDOS by introducing additional sites in the vacuum. Hopping parameters, taking the exponential decay into account, connect the atomic lattice sites and the vacuum sites. The hopping matrix element between adjacent vacuum sites leads to a free electron-like dispersion and the on-site energy models the vacuum barrier.

In the periodic TB model, the vacuum decay of the electronic states is taken into account by the exponential factor $\exp \left(-2 z \sqrt{ }\left(\left(2 m \varphi / \hbar^{2}\right)+\mathbf{k}_{\|}^{2}\right)\right)$, where $z$ is the distance from the surface, $\varphi$ is the work function and $\mathbf{k}_{\|}$is the Bloch vector ${ }^{28}$. Owing to the $\mathbf{k}_{\|}$dependence, the vacuum decay favours states in the vicinity of the $\bar{\Gamma}$ point of the $2 \mathrm{D}$ Brillouin zone, as shown in Fig. 4 .

\section{References}

31. Datta, S. Electronic Transport in Mesoscopic Systems (Cambridge Univ. Press, 1995). 